\title{
Bacillus polymyxa and its Bacteriophages
}

\author{
By A. E. FRANCIS AND JOAN E. RIPPON \\ Wellcome Research Laboratories, Beckenham, Kent
}

SUMMARY : Thirty strains of Bacillus polymyxa were isolated from soil, vegetation and water, and nine strains were received from other sources. At the time of isolation the new strains were of a white or sporogenous colony type, but rapidly produced asporogenous and rough variants. Four bacteriophages were isolated from a variant of a soil strain and from soil and sewage. These differed in host-strain specificity, particle size, heat resistance, citrate sensitivity and serological reactions.

Following the report by Ainsworth, Brown \& Brownlee (1947) of an antibiotic-'Aerosporin'-produced by Bacillus aerosporus (Greer), work was undertaken on certain aspects of the bacteriology of this organism. B. aerosporus is considered by Porter, McCleskey \& Levine (1937) to be identical with $B$. polymyxa, and on morphological, cultural, biochemical and serological grounds, we agree with this opinion.

Samples of soil, vegetation and water were obtained from widely separated localities in Great Britain, and these were cultivated by the methods described by Ledingham, Adams \& Stanier (1945). From these samples 30 strains of $B$. polymyxa were isolated. In addition, 4 strains were received from Dr Ainsworth's group, and 5 strains from other workers.

The main characters of this organism have been reported by Greer (1928), Porter et al. (1937) and Smith, Gordon \& Clark (1946). In the present paper it is proposed to record only certain morphological and colonial characters of the organism and the isolation and examination of four strains of bacteriophage active against it.

Colonial variation in $\mathbf{B}$. polymyxa

Ledingham et al. (1945) illustrated a bewildering variety of colony types of B. polymyxa. The description of the 'typical' colony by Smith et al. (1946) is undoubtedly that of the rough type and is typical of the two strains which we obtained from the National Collection of Type Cultures, No. 1380, and the American Type Culture Collection, No. 8523, whereas the colony described by Smith $e t$ al. as rough is the white or sporogenous parent colony.

Since the method of isolation employed included the pasteurization of soil samples before culture, the majority of strains were isolated as sporogenous colonies. Subsequent study has shown that these represent the parent colony type, since variants are thrown from them which have never reverted to the parent type.

Presumptive identification of colonies on the plating medium of Ledingham et al. (1945) was made on morphological grounds and the development of typical spores. Likely colonies were subcultivated in nutrient broth, and only those developing obvious ropiness were further examined. Final identification was 
made on biochemical grounds, and by bacteriophage and serological tests. The serological methods will be the subject of a later communication.

With freshly isolated strains, the typical colony developed in cultures on nutrient agar after $24 \mathrm{hr}$. incubation at $28^{\circ}$ is smooth, flat or low convex, circular, slightly brownish and about $1 \mathrm{~mm}$. in diameter. After $48 \mathrm{hr}$. it is 2-3 mm. in diameter, low convex, white and opaque with an entire edge. The $24 \mathrm{hr}$. culture consists of vegetative bacilli about $3 \times 1 \mu$, but at $48 \mathrm{hr}$. a large proportion of the bacilli are forming central or subterminal spores and have become spindle-shaped. After 3 days or longer, the culture consists mainly of oval spores, surrounded by a rim of stainable cytoplasm, which subsequently disappears.

When broth cultures of the white colonies are left at room temperature for a few days and then plated, it is often found that in addition to the white (sporogenous) colonies there are other colonies, which after 2 or 3 days' incubation remain brown, though of the same size as the white colonies (Pl. 1, fig. 1) These brown colonies consist of vegetative bacilli, many of which have undergone partial autolysis, and only occasionally can spores be found. These colonies do not revert to the white type.

When broth cultures of white or of brown colonies are left for 7 days or longer at room temperature, typical rough colonies also appear (PI. 1, fig. 2). These breed true and do not revert; when plated on soft or rather moist agar, they tend to swarm and to form a thin, grey sheet of growth.

A fourth or mucoid type of colony was also found in a few cultures of brown variants kept for many weeks in the cold room, but this type could not be isolated with any regularity. These colonies are much larger than the brown colonies, often $4 \mathrm{~mm}$. in diameter; after 2 or 3 days' incubation they are markedly umbilicated (Pl. 1, fig. 3) and are more glairy and sticky than the brown colonies. In stained films the appearance closely resembles that of the brown colony, showing vegetative bacilli, many partially autolysed bacilli and few spores; but individual bacilli tend to be smaller than those of the typical brown colony.

Sporogenous strains that have become largely replaced by brown or rough variants may be 'rejuvenated' either by heating broth cultures at $55^{\circ}$ or by prolonged storage of agar slope cultures in the cold room, even when white colonies cannot be demonstrated by direct plating.

It should be mentioned that different strains vary widely in the speed with which they produce variants. Some strains remain stable for many weeks at room temperature; others throw brown or rough variants which largely replace the parent colony type in a week or two at room temperature. Storage of cultures in the cold-room almost abolishes this variation. The amount of mucinous substance formed by different strains varies, and this modifies the colonial appearance to some extent. Rough variants produce less mucinous substance, both on plates and in nutrient broth, and much more is produced in media containing added carbohydrate. The exact nature of the mucinous substance has not been determined. 


\section{BACTERIOPHAGES FOR B. POLYMYXA}

Sources and methods of isolation

Four different strains of bacteriophage active against $B$. polymyxa were isolated.

Phage A was isolated (August, 1947) from culture-tanks in which B. polymyxa $\mathrm{CN} 1984^{*}$ was being grown for polymyxin production. This phage was subsequently propagated on a brown colony variant isolated from $\boldsymbol{B}$. polymyxa CN 1417, of which CN 1984 is a mucoid variant. CN 1417 was isolated from soil by Ainsworth et al. (1947).

Phages $\mathrm{B}$ and $\mathrm{C}$ were both isolated from a pool of soil samples during a search for a phage which would attack $\mathrm{CN} 2002$, a strain of $B$. polymyxa resistant to phage A. Nine samples of Sussex soils and waters were pooled after cultures for $B$. polymyxa had been prepared from them individually. Five of the soil samples subsequently yielded strains of $B$. polymyxa. The pool of soil samples was added to $100 \mathrm{ml}$. glucose phosphate peptone water (methyl red, Voges-

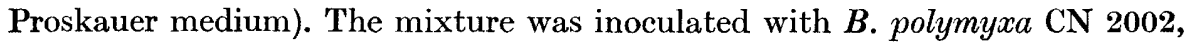
incubated for 2 days at $28^{\circ}$ and the culture filtered through paper and then through a Doulton porcelain filter-candle. The bacteria-free filtrate was propagated daily on $\boldsymbol{B}$. polymyxa $\mathrm{CN} 2002$ for one week, and the final culture filtered as before. Serial decimal dilutions spotted on an agar plate freshly inoculated with $B$. polymyxa $\mathrm{CN} 2002$ showed the presence of phage in high titre. This phage did not produce confluent lysis in the true sense, since a thin film of phage-resistant organisms was always found covering the area of lysis.

The above crude preparation was found to attack all the strains of $\boldsymbol{B}$. polymyxa available at that time. The appearance of the plaques varied on different strains of $B$. polymyxa, and grey haloes were often formed in the growth around the 'phaged' areas. A pure Phage B was isolated from a single plaque. Some strains of $\boldsymbol{B}$. polymyxa proved to be resistant to the action of this phage.

Phage $\mathrm{C}$ was isolated (March, 1948) from the same crude phage by picking a plaque from a plate spread with $B$. polymyxa $\mathrm{CN} 2366$, a strain resistant to phage $\mathbf{B}$. This phage $\mathrm{C}$, which was further purified, attacked almost all strains of $B$. polymyxa tested, and gave complete lysis of the host strain $\mathrm{CN} 2366$ on an agar plate culture.

Phage D was isolated (July, 1948) from sewage taken from the main drain of these laboratories. The sewage was diluted with an equal volume of glucose + phosphate + peptone water and divided into nine parts. Each of these samples was inoculated with a different strain of $B$. polymyxa and was incubated at $28^{\circ}$ overnight. After paper and filter-candle filtrations, the bacteriafree filtrates were inoculated into cultures of the respective host strains. After 24. hr. incubation five cultures showed good bacterial growth, but four remained clear and phages were isolated from these. The plaque appearances produced by the phages isolated from these four cultures corresponded with one another

* ' $\mathrm{CN}$ ' numbers are the culture numbers in the bacterial collection of the Wellcome Research Laboratories, Beckenham. 
on every strain of $\boldsymbol{B}$. polymyxa on which they were tested. They were therefore assumed to be identical, and the purified phage propagated on $\boldsymbol{B}$. polymyxa CN 2161 was labelled phage $D$.

\section{Activity of B. polymyxa phages}

The four phages differ in their host range among the strains of $B$. polymyxa (Pl. 2, figs. 1-4). Phage $C$ attacked all but one (CN 1576) of the strains against which it was tested. Phages A, B and D differ in the strains which they can attack. Table 1 shows the grouping of the B. polymyxa strains on the basis of their phage sensitivity. This grouping so far cannot be correlated with any other property of $B$. polymyxa.

Table 1. Distribution.table of sensitivity of strains of B. polymyxa to four types of bacteriophage

Strains of B. polymyxa (CN numbers)

$1417,1418,1971,2222,2225,2227,2228,2368,2490$

2224

$2161,2229,2369,2370,2492$

$1419,1951,1966,2002,2136,2159,2180,2184,2185$

2366

$2135,2163,2164,2183,2191$

$2181,2182,2401$

$2162,2179,2186,2220,2230,2491$

\begin{tabular}{cccc}
\multicolumn{5}{c}{ Sensitivity to phages } \\
A & B & C & D \\
+ & + & + & + \\
+ & + & + & - \\
+ & - & + & + \\
- & + & + & + \\
+ & - & + & - \\
- & + & + & - \\
- & - & + & + \\
- & - & + & -
\end{tabular}

These $B$. polymyxa phages have been tested against seventeen other members of the genus Bacillus. A single strain of each of the following species, classified according to Smith et al., was tested.

Group 1. B. megatherium, B. cereus, B. cereus var. mycoides, B. subtilis, B. subtilis var. aterrimus, B. subtilis var. niger, B. pumilus, B. coagulans, B. firmus.

Group 2. B. macerans, B. circulans, B. laterosporus.

Group 3. B. sphaericus, B. sphaericus var. rotans, B. sphaericus var. fusiformis.

None of these cultures was lysed by any of the four $B$. polymyxa phages.

Phage $\mathbf{A}$ is the most virulent of the four phages. It causes complete lysis on a plate and in liquid culture. Secondary growth is very rare and usually does not appear in liquid medium even on prolonged incubation. With all four phages the appearance of the plaques varies considerably with the strain of bacillus under test, and with the dryness and thickness of the agar. Phage $\mathbf{A}$ gives clear plaques, $\mathbf{0} \cdot \mathbf{5 - 2 . 0} \mathrm{mm}$. in diameter. The edges may be entire or with sharp indentations. Haloes up to $1 \mathrm{~cm}$. in diameter develop on keeping the plate at room temperature.

Phage B never gives complete lysis on a plate and resistant organisms always develop in liquid culture. On a plate culture of a susceptible strain, the area of lysis is always covered by a thin film of growth (Pl. 2, fig. 5). Individual plaques vary between 0.5 and $2.0 \mathrm{~mm}$. in diameter, and the edges are usually ill defined. Haloes are sometimes present, and are visible on plates when removed from the incubator. The bacteria which grow in the presence of phage B are 
resistant to it. Single colonies obtained by plating a broth culture of the growth scraped from the area of 'confluent lysis' were resistant to phage B. Filtrates of broth cultures grown from such colonies may contain a phage that resembles phage $B$ in its behaviour and is inhibited by an antiserum prepared against phage B. Phage has not been found in broth filtrates of normal strains.

Although phage $\mathrm{C}$ completely lyses the host strain $\mathrm{CN} 2366$ on solid medium, secondary growth may occur in liquid medium with some strains, but this is not constant. The plaques formed by the $\mathrm{C}$ phage on first isolation were very small, often invisible but for a large halo. However, after repeated culturing on strain $B$. polymyxa $\mathrm{CN} 2366$, the plaques increased in size, although their appearance remains very variable. We have evidence suggesting that propagation of phage $\mathbf{C}$ on different host strains results in changes in specificity in a manner analogous to that of Craigie's type II typhoid phage (Craigie \& Yen, 1938).

Some strains of $B$. polymyxa are completely resistant to phage $\mathbf{D}$ on solid medium, others are fully susceptible, or secondary growth may develop in the areas of lysis. In a liquid medium, growth always appears within $48 \mathrm{hr}$. even if the phage-treated culture remains clear after $24 \mathrm{hr}$. incubation. The plaques vary between 0.5 and $4 \mathrm{~mm}$. in diameter. Well-defined haloes are sometimes present, and with a few strains there is a well-marked white rim of increased bacterial growth around the area of lysis and within the halo.

\section{Inter-strain inhibition in B. polymyxa}

Early in work with phage A it was found that broth-filtrates of one strain of $\boldsymbol{B}$. polymyxa might inhibit the growth of another, and even produce an area of complete inhibition of growth, resembling phage lysis, when a drop of bacteria-free filtrate or of broth-culture was spotted on a plate spread with the second strain. At first this was thought to be due to phage, and that one strain was lysogenic for another. On dilution, however, the area of inhibition became fainter, and at dilutions greater than 1 in 100 it usually disappeared. True phage plaques were never demonstrated (Pl. 1, fig. 4). The antibiotic effect of strains of $B$. polymyxa upon one another has been reported by Katznelson (1944), who also encountered a bacteriophage for this species.

Strains of B. polymyxa differ, among other things, in the type of polymyxin produced: five polymyxins are now recognized (Brownlee \& Jones, 1948). It was thought that there might be some correlation between the inter-strain antibiotic effect and the polymyxin types involved. Accordingly all available strains of $B$. polymyxa were tested according to the method of Fisk (1942). A remarkable series of results was thus obtained. The patches of growth of the test cultures might merge into the growth of the underlying strain or they might be distinct from it but without inhibitory effect. Inhibition varying from a greyish ring to marked inhibition resembling phage lysis might be present (Pl. 1, fig. 5). A few strains inhibited themselves under these conditions, but only to a minor extent.

The strains could be grouped on the basis of the number of strains which they inhibited, and on their resistance to inhibition, but no useful classification 
resulted. Moreover, the type of polymyxin produced bore no relation to the antibiotic activity of any particular strain. The inhibitory material was produced more readily on solid medium than in broth. This inhibitory effect of one strain of $\boldsymbol{B}$. polymyxa upon another does not appear to be related to the substance produced by $B$. polymyxa which inhibits Staph. aureus (Stansly \& Schlosser, 1947). A strain which inhibits few other B. polymyxa strains will lyse Staph. aureus just as readily as one which is active against most other strains of its own species. Numerous attempts to isolate a phage from actively inhibitory strains have failed. Artificial lysogenic strains exist in the form of cultures of phage B-resistant organisms, but we have not encountered a naturally lysogenic culture.

\section{Differential properties of the bacteriophages}

In addition to the different lytic activities of the four phages, other properties were examined. Phage B is distinguished by its serological specificity, its large particle size, and its greater heat resistance relative to the other three phages. Phage $\mathbf{D}$ is citrate-resistant. Phages $\mathbf{A}$ and $\mathbf{C}$ are closely related and cannot be clearly separated by any of the tests employed, but three serial passages of each phage on the host strain for the other have not changed the strain specificity of either. All four phages are inhibited by the methylene-blue test and the urea test described by Burnet (1933b).

Particle size. By the use of collodion membranes prepared by the methods of Elford (1931) we made an approximate determination of the average particlesize of the four phages. The phage preparations, consisting of filtered brothcultures, were diluted with equal volumes of fresh nutrient broth, and filtered at $\mathrm{pH} 7 \cdot 5 \pm 0 \cdot 1$. All preparations were filtered through a membrane of average pore diameter $0.64 \mu$ before being subjected to a filtration test through membranes of smaller pore diameter. No attempt was made to determine the endpoint of filtration for any phage. The following results show the range between two membranes defining the phage size:

Phage $\mathbf{A}=33-60 \mathrm{~m} \mu$ : probably nearer the upper limit.

Phage $\mathrm{B}=60-113 \mathrm{~m} \mu$ : probably nearer the upper limit.

Phage $\mathrm{C}=33-83 \mathrm{~m} \mu$ : probably nearer the lower limit.

Phage $\mathbf{D}=21-83 \mathrm{~m} \mu$ : probably nearer the lower limit.

Phage $\mathbf{B}$ is thus distinguished from the other three phages by its markedly larger diameter.

Heat resistance. All four phages are remarkable for their heat lability. The results of heating suspensions of these phages in nutrient broth at $\mathrm{pH} \mathbf{7 \cdot 2}$ are shown in Table 2. Phage $B$ is again distinct, in that considerable activity remains after heating to $50^{\circ}$.

Growth rate. The final phage concentration"of all four phages when propagated on their respective host strains in nutrient broth and incubated at $28^{\circ}$ overnight is of the order of $10^{8}$ particles $/ \mathrm{ml}$. In $6 \mathrm{hr}$. phages $\mathrm{A}, \mathrm{C}$ and $\mathrm{D}$ will have almost reached this concentration, but phage $B$ multiplies much less rapidly. The rate of phage multiplication is much diminished at $37^{\circ}$ as compared with that at $28^{\circ}$, with all four phages. 
Counts of the number of phage particles liberated from one host cell have been made, by the method of Burnet (1929). With all four phages, the burstcount appears to be of the order of $2^{7}-2^{8}(128-256)$. The burst-time with phage $A$ is about 150 min., with phage $B$ somewhat over 360 min., with phage $C$ and

Table 2. Heat resistance of $\mathrm{B}$. polymyxa bacteriophages

Samples of phage suspensions were diluted 1 in 10 in nutrient broth at a final pH of $7 \cdot 2$. Five ml. volumes in $6 \times \frac{5}{8}$ in. test-tubes were heated for $20 \mathrm{~min}$. in water-baths at the required temperatures.

Counts given represent numbers of phage particles in $1 / 2000 \mathrm{ml}$. of the undiluted phage preparations. The figures for $45^{\circ}$ were obtained from a separate experiment.

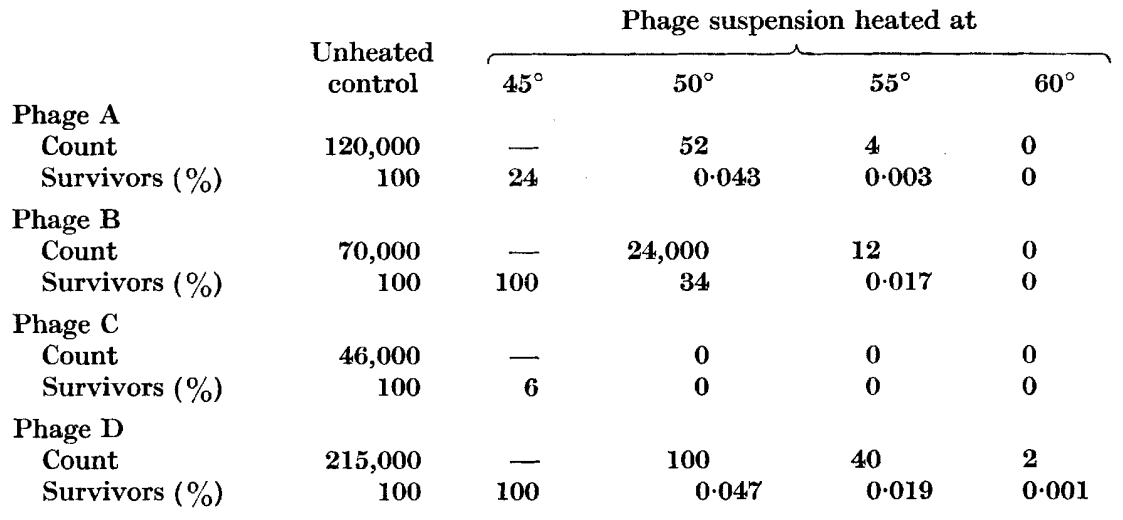

phage $\mathbf{D}$ about $200 \mathrm{~min}$. Confirmation of the first two figures has been obtained by another method. Plaque counts were made from phage preparations incubated with the host culture over a longer period (up to $8 \mathrm{hr}$.). The counts were plotted on a logarithmic scale and the time required for the count of phage particles to double at the maximum growth rate was read off. This gives a doubling division time or mean generation time of $18 \mathrm{~min}$. for phage $\mathbf{A}$ and of $45 \mathrm{~min}$. for phage $\mathrm{B}$. If the burst count is taken as $2^{8}$ for each phage, the mean generation times deduced from the first experiment would be $18 \frac{3}{4} \mathrm{~min}$. for phage $A$, somewhat over $45 \mathrm{~min}$. for phage $B$ and about $25 \mathrm{~min}$. for phages $\mathrm{C}$ and $\mathrm{D}$. In non-aerated nutrient broth the mean generation time for B. polymyxa at $28^{\circ}$ is a little over $1 \mathrm{hr}$.

Thus, phages $\mathbf{A}, \mathrm{C}$ and $\mathrm{D}$ multiply at much the same rate and have similar burst-times; phage $\mathbf{B}$ multiplies much more slowly and differs markedly from the other three.

Serological relationships. A method similar to that of Burnet (1933a) was used. Antisera were prepared by the injection of rabbits with filtered brothculture lysates. Serial dilutions of the antisera were mixed with equal volumes of the phage preparation under test, so diluted that $0.1 \mathrm{ml}$. of the mixture gave about 100 phage plaques. The mixtures were incubated at $37^{\circ}$ for $2 \mathrm{hr}$. and $0.1 \mathrm{ml}$. volumes then spread on an agar plate freshly inoculated with the host strain. Titres are recorded as the final dilution of the serum which gives a count amounting to $20 \%$ of the control count. The results of a typical experiment are shown in Table 3. 
Citrate sensitivity. This was determined by the method of Burnet (1933b), modified to give a roughly quantitative result. Serial tenfold dilutions of the phage preparation were dropped in $0.02 \mathrm{ml}$. quantities on nutrient agar plates to which had been added $0,0 \cdot 1,0.25,0.5,1.0$ and $1.5 \%$ sodium citrate, and

Table 3. Serological relationships of $\mathrm{B}$. polymyxa phages

\begin{tabular}{crrrr} 
Antiserum & \multicolumn{3}{c}{ Inhibitory titre of antiserum with phag } \\
\cline { 2 - 5 } to phage & $\mathrm{A}$ & $\mathrm{B}$ & $\mathrm{C}$ & $\mathrm{D}$ \\
A & 19,200 & 0 & 19,200 & 4,800 \\
B & 0 & 38,400 & 0 & 0 \\
C & 2,400 & 0 & 2,400 & 600 \\
D & 4,800 & 0 & 2,400 & 9,600
\end{tabular}

' 0 ' indicates no inhibition by the serum at a dilution of 1 in 300 .

which had been freshly inoculated with the appropriate strain of $B$. polymyxa. It was found that phages $\mathrm{A}$ and $\mathrm{C}$ were decreased to $10 \%$ of their initial activity by $0.25 \%$ citrate and almost inactivated by $0.5 \%$; phage $B$ was decreased to $10 \%$ of its initial activity by $0.5 \%$ citrate and almost inactivated by $1 \%$; phage $\mathrm{D}$ was unaffected by $1.5 \%$ citrate even though growth of the host strains was diminished by this concentration.

We are indebted to Dr C. L. Oakley for the collodion membranes and for advice on the technique of the phage filtrations. We wish to thank Mr E. A. Jones and Mr E. E. Hitchcock for the photographs, and Mr M. S. Beer and Mrs A. N. Cook for valuable technical assistance.

\section{REFERENCES}

Ainswonth, G. C., Brown, A. M. \& Brownlee, G. (1947). 'Aerosporin', an antibiotic produced by Bacillus aerosporus Greer. Nature, Lond., 160, 263.

Brownles, G. \& Jones, T. S. G. (1948). The polymyxins: a related series of antibiotics derived from $B$. polymyxa. Biochem. $J .43, \mathrm{xxv}$.

Burnet, F. M. (1929). A method for the study of bacteriophage multiplication in broth. Brit. J. exp. Path. 10, 109.

Bunnet, F. M. (1933a). The classification of dysentery-coli bacteriophages. II. The serological classification of coli-dysentery phages. J. Path. Bact. 36, 307.

Burnet, F. M. (1933b). The classification of dysentery-coli bacteriophages. III. A correlation of the serological classification with certain biochemical tests. J. Path. Bact. 37, 179.

Craigre, J. \& Yen, C. H. (1938). The demonstration of types of B. typhosus by means of preparations of Type II Vi phage. Canad. publ. Hlth J. 29, 448.

ELford, W. J. (1931). A new series of graded collodion membranes suitable for general bacteriological use, especially in filtrable virus studies. J. Path. Bact. 34, 505.

Fisk, R. T. (1942). Studies on Staphylococci. I. Occurrence of bacteriophage carriers among strains of Staphylococcus aureus. J. infect. Dis. 71, 153.

GreEr, F. E. (1928). The sanitary significance of lactose-fermenting bacteria not belonging to the $B$. coli group. I. Groups reported in the literature and isolated from water in Chicago. J. infect. Dis. 42, 501.

Katznelson, H. (1944). Bacteriophage of B. polymyxa in relation to 2, 3-butanediol fermentation. Canad. J. Res. (C), 22, 241. 
Journal of General Microbiology, Vol. 3, No. 3
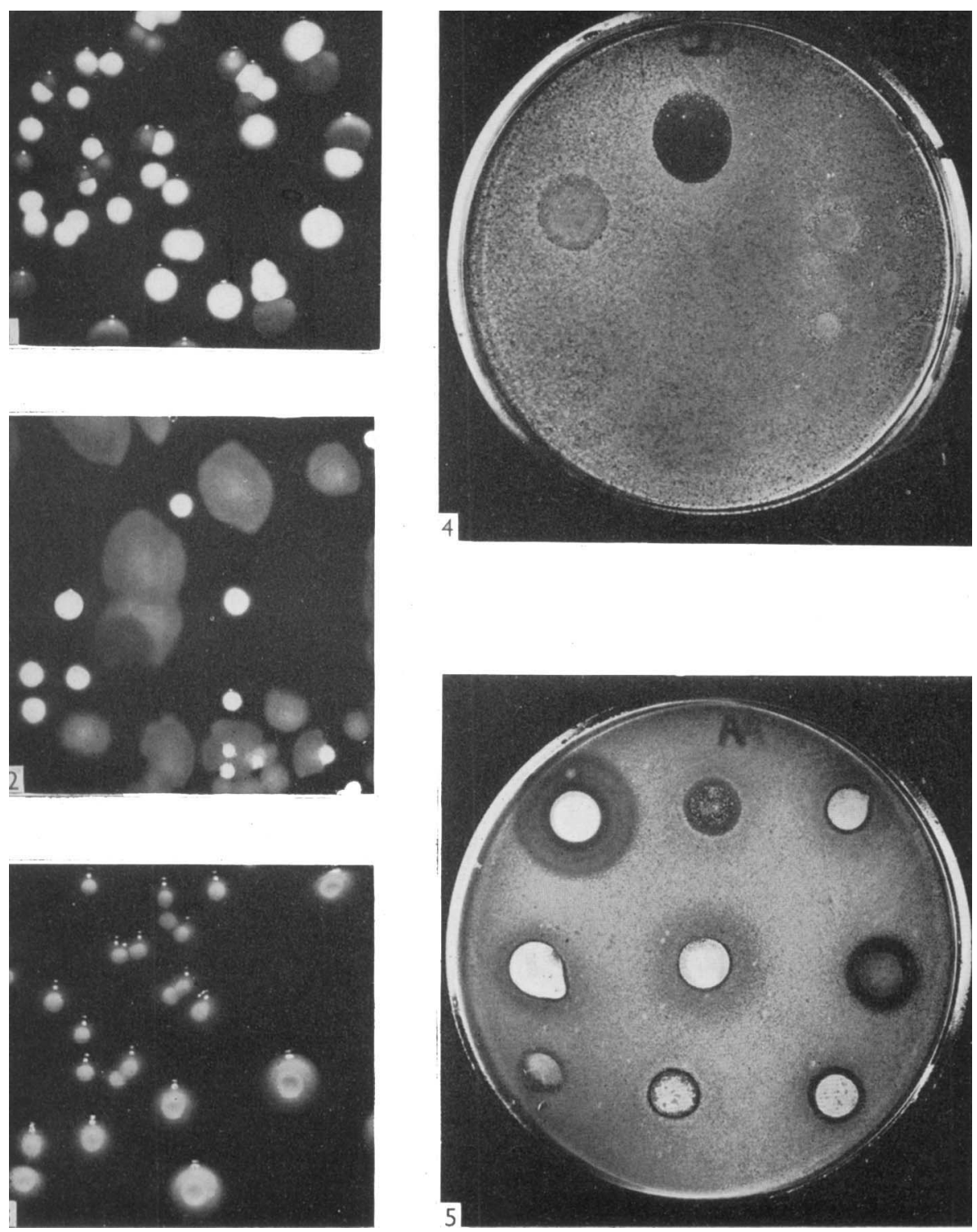

Figs. 1-5

A. E. Francis \& J. F. Rippon-Baclllus polymyxa. Plate 1 
Journal of General Microbiology, Vol. 3, No. 3

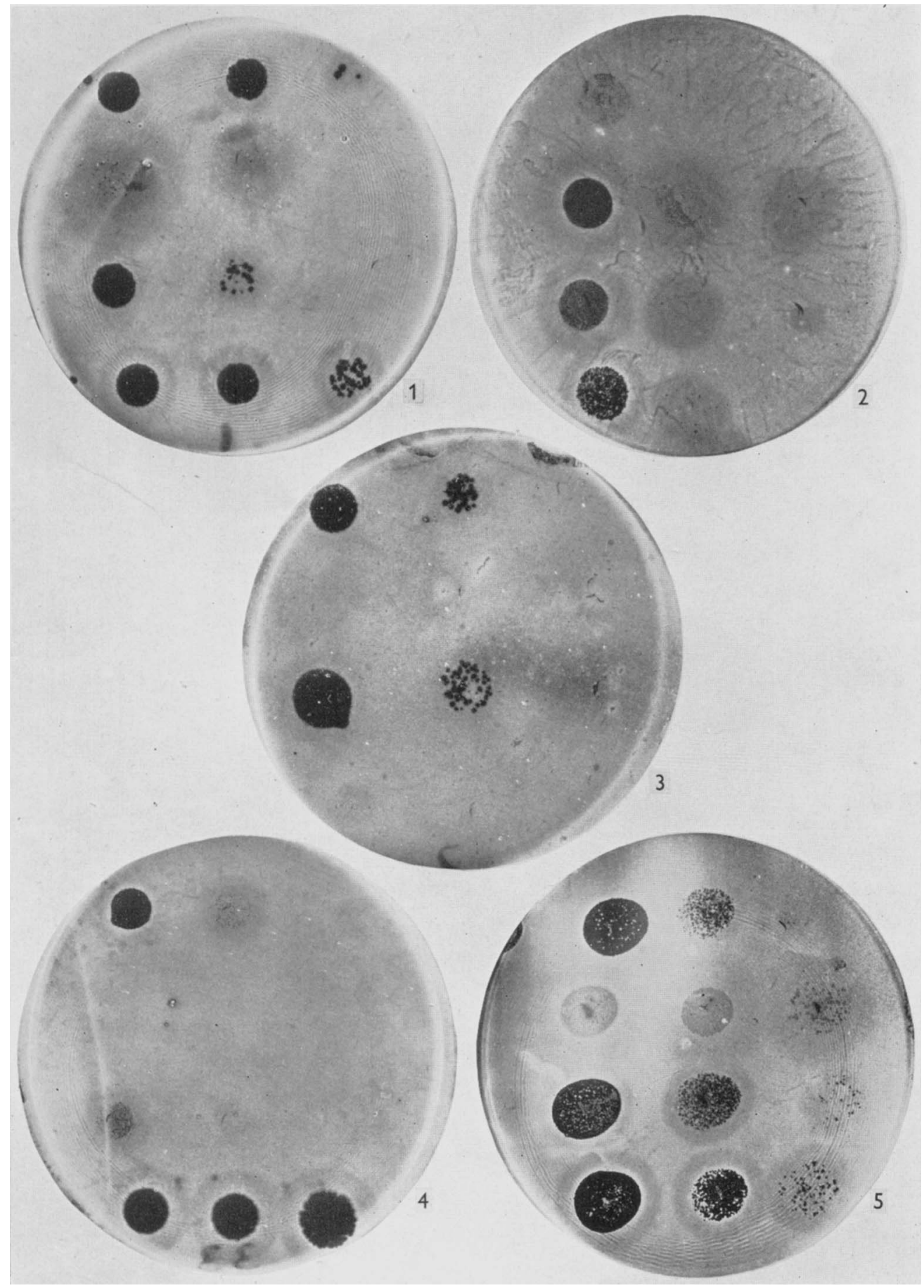

ligs. 1-5

A. E. Francis \& J. E. Rupron-Bachlhes polymyda. Piate 2 
Ledingham, G. A., Adams, G. A. \& Stanier, R. Y. (1945). Fermentation of wheat mashes by Aerobacillus polymyxa. Canad. J. Res. (F), 23, 48.

Porter, R., McCleskey, C. S. \& Levine, M. (1937). The facultative sporulating bacteria producing gas from lactose. J. Bact. 33, 163.

Smith, N. R., Gordon, R. E. \& Clark, F. E. (1946). Aerobic mesophilic sporeforming bacteria. Misc. Publ. U.S. Dep. Agric. No. 559. Washington, D.C.

Stansly, P. G. \& Schlosser, M. E. (1947). Studies on polymyxin. II. Isolation and identification of Bacillus polymyxa and differentiation of polymyxin from certain known antibiotics. J. Bact. 54, 549.

\section{EXPLANATION OF PLATES}

\section{Plate 1}

Figs. 1, 2 and 3. Magnification $\times 2$.

Fig. 1. B. polymyxa $\mathrm{CN} 1417$ showing white and brown colonies.

Fig. 2. B. polymyxa CN 1966 showing white and rough colonies.

Fig. 3. B. polymyxa $\mathrm{CN} \mathrm{1417,} \mathrm{mucoid} \mathrm{variant.}$

Fig. 4. Inter-strain inhibition. Filtrate of broth culture of B. polymyxa CN 1417 spotted on $B$. polymyxa CN 2002 in serial decimal dilutions, anticlockwise.

Fig. 5. Inter-strain inhibition. Plate spread with strain B. polymyxa CN 1966. Loopsful of broth cultures of nine other strains spotted on the plate. Incubated 2 days at $28^{\circ}$.

\section{Plate 2}

All figures show plate cultures of host strains of B. polymyxa with phages A, B, C and D arranged vertically; at dilutions of $1 / 10,1 / 10^{3}$ and $1 / 10^{5}$ from left to right.

Fig. 1. CN 1417, original host strain for phage A. Shows reaction with phages A, B, C and D.

Fig. 2. CN 2002, original host strain for phage B. Shows reaction with phages B, C and D; also antibiotic inhibitory effect with phage A preparation.

Fig. 3. CN 2366, original host strain for phage C. Shows reaction with phages $\mathrm{A}$ and $\mathrm{C}$.

Fig. 4. CN 2161, original host strain for phage D. Shows reaction with phages A, C and D.

Fig. 5. CN 2222. Shows reaction with phages A, B, C and D, with film of resistant organisms across areas of lysis with phage B. (Owing to the mucinous nature of broth cultures of B. polymyxa it is difficult to prevent the growth of colonies in what should be areas of confluent lysis, as with phages $\mathrm{A}, \mathrm{C}$ and $\mathrm{D}$ in this example.) 